\title{
Gravity changes and deformation due to a magmatic intrusion in a two-layered crustal model
}

\author{
José Fernández \\ Instituto de Astronomía y Geodesia, Facultad de Ciencias Matemáticas, Ciudad Universitaria, Madrid \\ John B. Rundle \\ Earth Sciences Department, Lawrence Livermore National Laboratory, University of California, Livermore
}

\begin{abstract}
We develop and extend theoretical and computational methods for the calculation of the deformation, gravity and potential change due to a point source of magma injection into a multilayered, elastic-gravitational earth model. In our calculations, which are based upon the method outlined by Rundle, two distinct layers overlying a half-space may be incorporated. The source can be located in either of the layers or the half-space. The method is quite general, and can be readily adapted to calculations in which stresses in either the layers or the half-space relax by viscoelastic flow. The results obtained indicate that the use of homogeneous half-space to represent the Earth may in some cases be too simple a model and that variations in elastic moduli have a more significant effect than variation in reference density on both the surface displacements and gravity changes. As an example, we calculate the displacement and gravity changes due to a subsurface mass injection in a crust-mantle model appropriate to the volcanic island of Lanzarote, which is presently the subject of numerous geophysical experiments. Both historical and recent data indicate that Lanzarote may be subject to some risk of volcanic eruption in the future, thus our calculations may be useful in interpreting observations of preeruption phenomena. The results are discussed in terms of prediction versus measurement capabilities.
\end{abstract}

\section{Introduction}

There are two fundamental and equally important aspects for understanding crustal deformation in volcanic regions: theoretical modeling of the effects caused by the natural phenomenon under study, in this case a magmatic intrusion in the crust; and the practical aspect of continuous observation and appropriate processing of the data obtained. The latter must be performed not only in areas of activity but also in areas of possible activity.

There is a very extensive literature on deformation modeling, most of which does not consider the existence of the ambient gravitational field, the crust being represented by a homogeneous, purely elastic half-space. Rundle [1980, 1982] obtains and solves the equations that represent the coupled elastic-gravitational problem for a stratified half-space of homogeneous layers, using the propagator matrix technique [Thomson, 1950; Haskell, 1953; Gilbert and Backus, 1966] to obtain the surface solutions (potential and gravity changes and deformation). Rundle [1981] develops the numerical formulation needed to compute these perturbations for the case of a single layer in welded contact with an infinite

\footnotetext{
${ }^{1}$ Now at Cooperative Institute for Research in Environmental Sciences, University of Colorado, Boulder, Colorado.

Copyright 1994 by the American Geophysical Union.

Paper number 93JB02449.

0148-0227/94/93JB-02449\$05.00
}

half-space. In the work by Rundle [1982] this model is used to model data from the posteruption deflation of Kilauea volcano in 1975 .

If we identify the mantle with the infinite half-space, the use of a homogeneous layer to represent the Earth's crust may in some cases be too simple a model. The possibility of developing crustal models with more than one layer has induced us to develop the numerical formulation necessary to obtain explicit expressions for solutions in the case of two layers overlying an infinite half-space. In the following sections, a description is given of the theoretical approach, followed by an extension to the case of a two-layered medium (lithosphere) overlying an infinite half-space (asthenosphere). By way of example, this formulation is applied to the crustal model available for the island of Lanzarote (Canary Islands).

\section{Presentation of the Problem}

A magmatic intrusion in the Earth's crust will cause a series of effects related to its mass as well as to the pressurization of the chamber due to overfilling or temperature changes. The effects of most interest for deformation modeling and prediction are those which in principle could be detected on the surface before the eruption, including surface gravity changes and deformation. Hence the problem lies in calculating the changes in the gravitational potential and gravity, and the deformation (vertical and horizontal) caused on the Earth's surface by a magmatic intrusion in the crust.

We consider a model of the Earth including an ambient 
gravitational field. To obtain the deformation, potential, and gravity changes, we solve the partial differential equations for a layered half-space that represents the Earth's crust and mantle. This half-space is an elastic medium where we place a source of perturbation, a spherical magmatic intrusion, which we treat as a point source.

Our model consists of a stratified half-space of $p-1$ isotropic and homogeneous layers (Figure 1) where the layers are numbered serially, the shallowest being 1 . The half-space is designated layer $p$. The intrusion is located at $(0,0, h)$.

\section{Solution}

The generalized static Navier equations which couple elastic and gravitational effects, satisfied by the displacement vector $\mathbf{u}$ and the perturbation potential $\phi$ in an elastic, self-gravitating uniform, infinite medium [Love, 1911] are

$$
\begin{gathered}
\nabla^{2} u+\frac{1}{1-2 \sigma} \nabla \nabla \cdot u+\frac{\rho_{0} g}{\mu} \nabla\left(u \cdot e_{z}\right)-\frac{\rho_{0}}{\mu} \nabla \phi-\frac{\rho_{0} g}{\mu} e_{z} \nabla \cdot u=0 \\
\nabla^{2} \phi=-4 \pi \rho_{0} G \nabla \cdot u
\end{gathered}
$$

In (1) and (2), $\phi$ is the gravitational potential (perturbation potential) produced by the intrusion, $\mathbf{u}$ is the displacement caused by this intrusion, $\rho_{0}$ is the constant unperturbed density, $G$ is the gravitational constant, $g$ is the unperturbed surface gravitational acceleration, $\sigma$ is the Poisson ratio and $\mu$ is the rigidity.

Using the method of Singh [1970], Rundle [1980, 1982] solved (1) and (2) in an infinite medium, and used the solutions obtained to generate solutions for a layered medium, by use of the propagator matrix technique. The solution in the layered medium takes the form

$$
\begin{gathered}
u=M \int_{0}^{\infty}\left[x_{0}^{1}(0) P_{0}+y_{0}^{1}(0) B_{0}\right] k d k \\
\delta g=-\frac{d \phi}{d z}=-M \int_{0}^{\infty} q_{0}^{1}(0) J_{0}(k r) k d k+\beta_{0} u_{z}
\end{gathered}
$$

where $M$ is the mass of the intrusion and $x_{0}{ }^{1}(0), y_{0}{ }^{1}(0)$ and $q_{0}{ }^{1}(0)$ are the integration kernels which we will obtain further on. $\mathbf{P}_{0}$ and $\mathbf{B}_{0}$ are given in terms of the Bessel function of the first kind of order zero $J_{0}(k r)$ (equation (6) of Rundle [1982]), and $\beta_{0}=4 \pi G \rho_{0}$ where $\rho_{0}$ is the density of layer 1. A uniqueness theorem can be demonstrated for this solution [Rundle, 1982].

\section{Integration Kernels}

The integration kernels $x_{0}^{1}(0), y_{0}^{1}(0)$ and $q_{0}^{1}(0)$ are given as a function of the characteristics of the medium's layers, through matrix [E] and a vector [F] of the form

$$
\begin{aligned}
& x_{0}^{1}(0)=E_{11} A_{0 p}+E_{13} B_{0 p}+E_{15} D_{0 p}-F_{1}, \\
& y_{0}^{1}(0)=E_{21} A_{0 p}+E_{23} B_{0 p}+E_{25} D_{0 p}-F_{2}, \\
& q_{0}^{1}(0)=E_{61} A_{0 p}+E_{63} B_{0 p}+E_{65} D_{0 p}-F_{6} .
\end{aligned}
$$

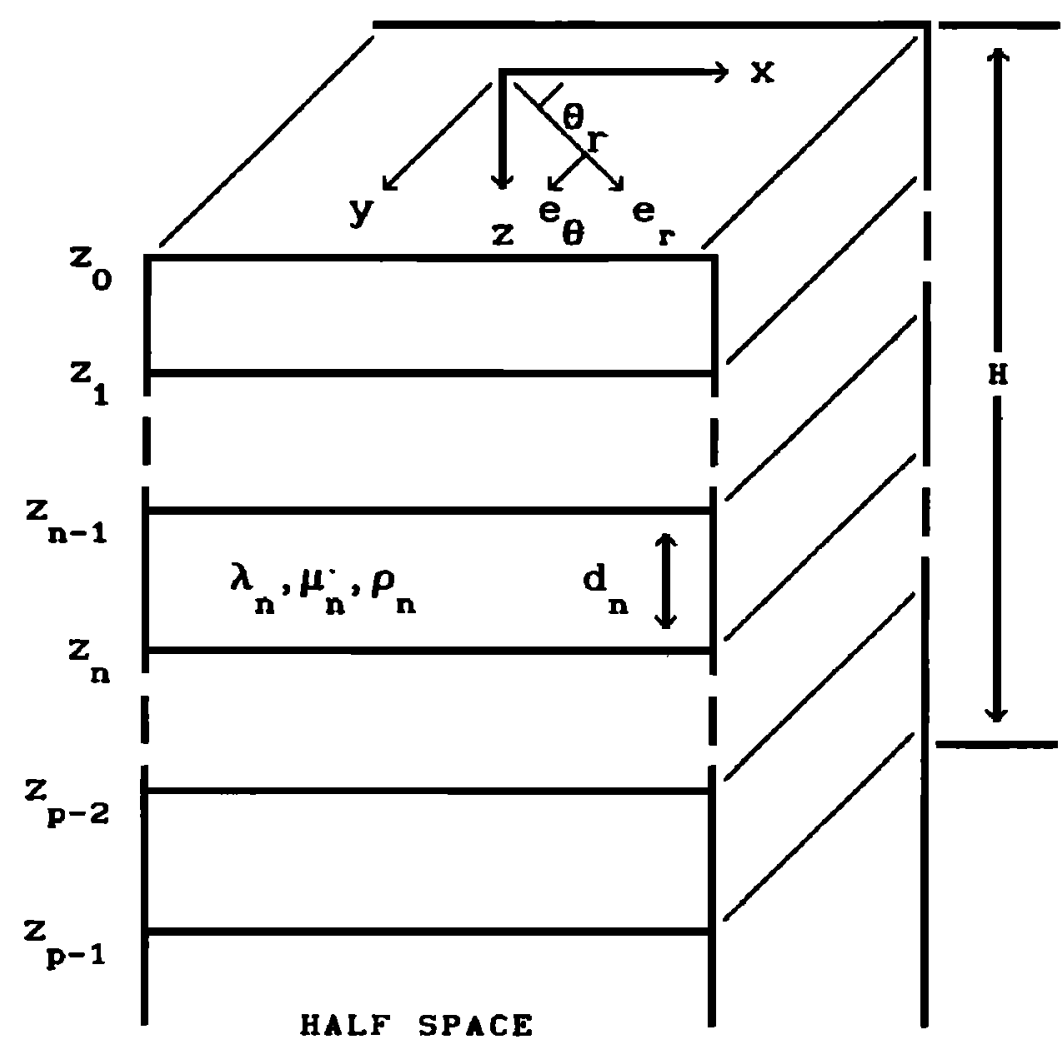

Figure 1. Layered half-space. 
The matrix [E], a product of propagator matrices (layer matrices), is obtained using the solutions of the infinite space problem [Rundle, 1980]. Vector [F] is a product of layer matrices with the jump discontinuity vector [D]. Matrix [E] is given by

$$
[E]=\left[a^{1}\right] \cdot\left[a^{2}\right] \cdots\left[a^{p-1}\right] \cdot\left[Z^{p}(H)\right]
$$

while $\left[a^{n}\right]$ is the layer matrix (propagator matrix) defined as

$$
\left[a^{n}\right]=\left[Z^{n}\left(-d_{n}\right)\right] \cdot\left[Z^{n}(0)\right]^{-1} \text {. }
$$

The elements of the matrix $\left[Z^{n}(z)\right]$ are functions of $k$ given by Rundle [1980, 1982] and Fernández [1992].

Vector [F] is defined by the product

$$
[F]=\left[a^{1}\right] \cdot\left[a^{2}\right] \cdots\left[a^{s l}\right] \cdot[D] .
$$

A new layer boundary is introduced at depth $h$ corresponding to the depth of the intrusion $\left(z_{s I}=h\right)$, and $s 1$ corresponds to the division of the layer where the intrusion is located.

[D] is the jump discontinuity vector for $z=h$. With these definitions, the coefficients $A_{0 p}, B_{0 p}$ and $D_{0 p}$ take the form

$$
\begin{aligned}
A_{0 p}=[ & \left(k F_{5}-F_{6}\right) E_{35}^{34}+\left(k E_{53}-E_{63}\right) E_{F 5}^{43} \\
& \left.+\left(k E_{55}-E_{65}\right) E_{F 3}^{34}\right] \cdot \Delta^{-1} ; \\
B_{0 p}= & {\left[\left(k E_{51}-E_{61}\right) E_{F 5}^{34}+\left(k F_{5}-F_{6}\right) E_{15}^{43}\right.} \\
& \left.+\left(k E_{55}-E_{65}\right) E_{F 1}^{43}\right] \cdot \Delta^{-1} ; \\
D_{0 p}=[ & \left(k E_{51}-E_{61}\right) E_{F 3}^{43}+\left(k E_{53}-E_{63}\right) E_{F 1}^{34} \\
& \left.+\left(k F_{5}-F_{6}\right) E_{13}^{34}\right] \cdot \Delta^{-1} ;
\end{aligned}
$$

with

$$
\begin{gathered}
\Delta=\left(k E_{51}-E_{61}\right) E_{35}^{34}+\left(k E_{53}-E_{63}\right) E_{15}^{43} \\
+\left(k E_{55}-E_{65}\right) E_{13}^{34}, \\
E_{k l}^{i j}=E_{i k} E_{j l}-E_{i l} E_{j k}, \\
E_{F k}^{U j}=F_{i} E_{j k}-F_{j} E_{i k}
\end{gathered}
$$

\section{Jump Discontinuity Vector}

For a magmatic intrusion, considered as a point mass $M$ placed suddenly at a depth $h$, the source functions (jump discontinuities) would be those resulting from the interaction between the mass $M$ and the ambient gravity field (14) and (15); and those which reflect the effects caused by the pressurization of the magmatic chamber due to overfilling or temperature changes (16) and (17) [Rundle, 1980, 1982]:

$$
\begin{aligned}
& D_{3}=\frac{-M g}{4 \pi k} \\
& D_{6}=2 G M
\end{aligned}
$$

$$
D_{1}=\frac{2 p a^{3}(1-\sigma) \delta}{\mu(1+\delta)}
$$

$$
D_{4}=\frac{2 p a^{3}(1-\sigma) \delta}{1+\delta}
$$

where $p$ is the pressure in the magmatic chamber, $a$ its radius and $\delta=1 /(3-4 \sigma)$.

\section{Gravity Changes}

In a nonoceanic Earth model, we need expressions that will give us the surface, free air and Bouguer gravity changes, which will be those observed directly or determined from the observed data. The expressions for any vertical displacement $u_{z}$, obtained by Rundle [correction to his 1982 paper], are as follows:

Surface gravity change

$$
g_{s}=-\frac{\partial \phi}{\partial z}-4 \pi \rho G u_{z}+\gamma_{F A} u_{z}
$$

Free Air gravity change

$$
g_{F A}=-\frac{\partial \phi}{\partial z}-4 \pi \rho G u_{z}
$$

Bouguer gravity change

$$
g_{B}=g_{F A}+2 \pi p G u_{z}
$$

the quantity $\gamma_{F A}(=0.3085 \mathrm{mGal} / \mathrm{m})$ being the free air gradient. In (20)-(22) $\partial \phi / \partial z$ represents its value for $z=0$.

\section{Explicit Solutions for a Two-Layer Stratified Half-Space}

The model described has been applied by Rundle to a layered medium consisting of a layer overlying an infinite half-space [Rundle, 1982]. As mentioned above, the use of a single layer to represent the lithosphere may be too simple a model. In the present case it makes more sense to introduce layering into the elastic lithosphere, the shallowest layer representing oceanic crust, and the bottom layer representing the mantle component of the lithosphere.

We see [e.g., Rundle, 1978, 1981] that matrix $\left[Z^{\mathbf{n}}(\mathbf{z})\right]$ can be expressed as

$$
\left[Z^{n}(z)\right]=\left(\left[A^{n}(z)\right]+\left[B^{n}(z)\right] e^{-2 k z}\right) e^{k z}
$$

the elements of matrices $\left[\mathbf{A}^{\mathbf{n}}(\mathbf{z})\right]$ and $\left[\mathbf{B}^{\mathrm{n}}(\mathbf{z})\right]$ as given by Fernández [1992]. Matrix [ $\left.\mathbf{a}^{\mathrm{n}}\right]$ may be expressed in a similar fashion, using (9), 


$$
\left[a^{n}\right]=\left(\left[M^{n}\right]+\left[N^{n}\right] e^{-2 k d_{n}}\right) e^{k d_{n}}
$$

We must also obtain matrix [E] and the source functions vector $[\mathbf{F}]$ necessary for our calculations. For a two-layered half-space, matrix $[E]$ will be

$$
[E]=\left[a^{1}\right]\left[a^{2}\right]\left[Z^{3}(H)\right],
$$

which, considering (21) and (22) is given by

$$
[E]=\sum_{s=1}^{7}\left[E^{s}\right] e^{Z_{s}},
$$

with $Z_{1}=0, Z_{2}=-2 k d_{1}, Z_{3}=-2 k d_{2}, Z_{4}=-2 k H, Z_{5}=-2 k\left(H+d_{1}\right)$, $Z_{6}=-2 k\left(H+d_{2}\right)$ and $Z_{7}=-4 k H . E_{i j}{ }^{s}$ represents the element of row $i$, column $j$ of matrix $\left[\mathbf{E}^{\mathrm{s}}\right]$.

The expression of vector [F] will depend on the layer where the intrusion is located. Let us examine three possible cases.

\section{Intrusion in Layer 1}

Using (8) and (22), we have

$$
[F]=\sum_{s=1}^{2}\left[F_{1}^{s}\right] e^{D l},
$$

where $D 1_{1}=k h, D 1_{2}=-k h .\left(F_{1}\right)_{i}$ denotes the $i$ th element of vector $\left[\mathbf{F}_{1}^{\mathbf{s}}\right]$.

Intrusion in Layer 2

$$
[F]=\sum_{s=1}^{4}\left[F_{2}^{s}\right] e^{D 2_{s}},
$$

with $D 2_{1}=k h, D 2_{2}=-k\left(h-2 d_{1}\right), D 2_{3}=-k\left(2 d_{1}-h\right), D 2_{4}=-k h$.

Intrusion in Layer 3 (Half-Space)

$$
[F]=\sum_{s=1}^{8}\left[F_{3}^{s}\right] e^{D 3_{s}},
$$

where $\quad D 3_{1}=k h, \quad D 3_{2}=-k(h-2 H), \quad D 3_{3}=-k\left(2 d_{1}-h\right)$, $D 3_{4}=-k\left(h-d_{2}\right), \quad D 3_{5}=-k\left(2 d_{2}-h\right), \quad D 3_{6}=-k\left(h-2 d_{1}\right)$, $D 3_{7}=-k(2 H-h), D 3_{8}=-\mathrm{k} h$.

To calculate the kernel functions we will need to know $E_{k l}^{i j}$ and $E_{F k}^{i j}$, defined in (13). Utilizing (24), we can express $E_{k l}{ }^{i j}$ as a sum of products of $k$ functions multiplied by exponentials as

$$
E_{k l}^{i j}=e^{4 k H}\left(\sum_{s=1}^{19} E_{k l, j, j}^{s} e^{G_{s}}\right)
$$

The functions $E_{k l, i j}^{s}$ and exponents $G_{s}$ are given by Fernández [1992].

Using (13) and (24)-(27), we can express $E_{F k}^{i j}$ similarly, with the intrusion in
Layer 1

$$
E_{F k}^{i j}=e^{2 k H}\left(\sum_{s=1}^{14} E_{F k, i}^{s} e^{T l_{s}}\right)
$$

Layer 2

$$
E_{F k}^{i j}=e^{2 k H}\left(\sum_{s=1}^{20} E_{F k, i j}^{s} e^{\pi_{s}}\right),
$$

Layer 3 (half-space)

$$
E_{F k}^{i j}=e^{2 k H}\left(\sum_{s=1}^{28} E_{F k, j}^{s} e^{T 3_{s}}\right)
$$

The functions $E_{F k, i j}^{s}$ and the exponents $T R_{s}(R=1,2,3$; depending on the layer) are listed by Fernández [1992].

We also need to find expressions for $\left(k E_{5 i}-E_{6 i}\right)$ and $\left(k F_{5}-F_{6}\right)$. We have

$$
k E_{5 i}-E_{6 i}=e^{2 k H}\left(\sum_{s=1}^{7} E_{56 i}^{s} e^{Z_{s}}\right)
$$

with

$$
\begin{gathered}
E_{56 i}^{s}=k E_{5 i}^{s}-E_{6 i}^{s}, \\
k F_{5}-F_{6}=\sum_{s=1}^{N} F_{r 56}^{s} e^{D r_{s}},
\end{gathered}
$$

where $r$ is the number of the layer where the intrusion is located $(r=1,2,3), N$ varies, depending on the layer, between values 2,4 and 8 , respectively, and

$$
F_{r 56}^{s}=k\left(F_{r}^{s}\right)_{5}-\left(F_{r}^{s}\right)_{6}
$$

Utilizing (24)-(35) we calculate $A_{03}, B_{03}, D_{03}$, with which we can now obtain the kernels we need. The expressions for $A_{03}, B_{03}, D_{03}$, depend on the location of the intrusion. If located in layer 1 they are given by

$$
\begin{aligned}
& A_{03}=e^{4 k H}\left[\left(\sum_{s=1}^{2} F_{156}^{s} e^{D l_{s}}\right)\left(\sum_{m=1}^{19} E_{35,34}^{m} e^{G_{m}}\right)\right. \\
& +\left(\sum_{q=1}^{7} E_{563}^{q} e^{z_{q}}\right)\left(\sum_{r=1}^{14} E_{F 5,43}^{r} e^{T l_{r}}\right) \\
& \left.+\left(\sum_{u=1}^{7} E_{565}^{u} e^{Z_{u}}\right)\left(\sum_{v=1}^{14} E_{F 3,34}^{v} e^{T I_{v}}\right)\right] \cdot \Delta^{-1}, \\
& B_{03}=e^{4 k H}\left[\left(\sum_{s=1}^{2} F_{156}^{s} e^{D l_{s}}\right)\left(\sum_{m=1}^{19} E_{15,43}^{m} e^{G_{m}}\right)\right. \\
& +\left(\sum_{q=1}^{7} E_{561}^{q} e^{Z_{q}}\right)\left(\sum_{r=1}^{14} E_{F 5,34}^{r} e^{T l_{r}}\right) \\
& \left.+\left(\sum_{u=1}^{7} E_{565}^{u} e^{Z_{v}}\right)\left(\sum_{v=1}^{14} E_{F 1,43}^{v} e^{T 1_{v}}\right)\right] \cdot \Delta^{-1},
\end{aligned}
$$




$$
\begin{aligned}
D_{03}= & e^{4 k H}\left[\left(\sum_{s=1}^{2} F_{156}^{s} e^{D I_{s}}\right)\left(\sum_{m=1}^{19} E_{13,34}^{m} e^{G_{m}}\right)\right. \\
& +\left(\sum_{q=1}^{7} E_{561}^{q} e^{Z_{q}}\right)\left(\sum_{r=1}^{14} E_{F 3,43}^{r} e^{T I_{r}}\right) \\
& +\left(\sum_{u=1}^{7} E_{563}^{u} e^{Z_{v}}\right)\left(\sum_{v=1}^{14} E_{F 1,34}^{v} e^{T I_{v}}\right] \cdot \Delta^{-1}
\end{aligned}
$$

The value $\Delta$ which forms the denominator of the three previous terms takes the form

$$
\begin{aligned}
\Delta= & e^{6 k H}\left[\left(\sum_{s=1}^{7} E_{561}^{s} e^{Z_{s}}\right)\left(\sum_{m=1}^{19} E_{35,34}^{m} e^{G_{m}}\right)\right. \\
& +\left(\sum_{q=1}^{7} E_{563}^{q} e^{Z_{q}}\right)\left(\sum_{r=1}^{19} E_{15,43}^{r} e^{G_{v}}\right) \\
& \left.+\left(\sum_{u=1}^{7} E_{565}^{u} e^{Z_{u}}\right)\left(\sum_{v=1}^{19} E_{13,34}^{v} e^{G_{v}}\right)\right] .
\end{aligned}
$$

To obtain the expressions for layers 2 and 3 , we substitute in (36)-(38) for the sums in $F_{156}^{s}$ and $E_{F k, i j}^{s}$ the quantities

$$
\sum_{s=1}^{4} F_{256}^{s} e^{D 2}, \sum_{s=1}^{20} E_{F k, j}^{s} e^{T 2}
$$

for layer 2 , and

$$
\sum_{s=1}^{8} F_{356}^{s} e^{D 3}, \sum_{s=1}^{28} E_{F k, j}^{s} e^{T 3}
$$

for layer 3.

We can use these expressions to calculate the kernel functions we need: $x_{0}{ }^{1}, y_{0}{ }^{1}, q_{0}{ }^{1}$.

Using

$$
\tau=\Delta / e^{6 k H},
$$

for an intrusion located in the first layer, kernel $x_{0}{ }^{1}(0)$ will take the form

$$
\begin{aligned}
x_{0}^{1}(0)=\frac{1}{\tau} & {\left[\sum_{t=1}^{7} e^{Z_{t}}\left(E_{11}^{t} A_{03}+E_{13}^{t} B_{03}+E_{15}^{t} D_{03}\right)\right.} \\
& \left.-\sum_{m=1}^{2} \tau\left(F_{1}^{m}\right)_{1} e^{D 1_{m}}\right]
\end{aligned}
$$

where element $F_{1}$ appears multiplied and divided by $\tau ; A_{03}$, $B_{03}$ and $D_{03}$ given by equations (36)-(38).

To calculate $y_{0}^{1}(0)$ we change $E_{11}^{t}, E_{13}{ }^{t}, E_{15}{ }^{t},\left(F_{1}^{m}\right)_{1}$ to

$$
E_{21}^{t}, E_{23}^{t}, E_{25}^{t},\left(F_{1}^{m}\right)_{2}
$$

in (43); and to obtain $q_{0}{ }^{1}(0)$ we substitute into (44) with

$$
E_{61}^{t}, E_{63}^{t}, E_{65}^{t},\left(F_{1}^{m}\right)_{6}
$$

For an intrusion located in layer two we will now substitute the expressions for $A_{03}, B_{03}, D_{03}$, using (40); and (41) for an intrusion in layer three (half-space). The sum of functions that multiply $e^{k h}$ must be zero for convergence in (43), and we take advantage of this by setting them equal to zero.

In practice, (43) allows us to develop an easy to program computing algorithm, and the same occurs when we make the successive substitutions needed to calculate the various kernels.

\section{Testing of the Model}

Using the explicit solutions, we can compute the deformation and gravity change due to a magma intrusion. We are interested in distinguishing the effects of changes in layer densities and Lamé constants from the effects caused by the source. To examine these effects, we carried out calculations using a medium consisting of two layers, each with a nominal thickness of $10 \mathrm{~km}$, overlying a half-space. The top layer (layer 1) has reference density $\rho_{1}$ and Lamé constants $\mu_{1}, \lambda_{1}$, the middle layer has reference density and Lamé constants $\rho_{2}, \mu_{2}, \lambda_{2}$, and the half-space has reference density and Lamé constants $\rho_{3}, \mu_{3}, \lambda_{3}$. As a check on our calculations, we compare results to those obtained by placing similar sources in an elastic half space, and in a medium comprising of one layer $20 \mathrm{~km}$ thick overlying a homogeneous halfspace. Densities and Lamé constants for the various calculations are listed in Table 1.

Figure 2 shows the surface vertical and radial deformation, as well as the surface gravity change due to the presence of a center of expansion of strengh $\mathrm{pa}^{3}$ at $5 \mathrm{~km}$ depth (layer 1), where $p$ is the pressure in bars $\left(10^{5} \mathrm{~Pa}\right)$ and $a$ is the radius of the source in kilometers. It should be recalled that our calculations represent the lowest-order term in a multipole expansion of a more realistic spatially extended source. Reference to Table 1 should be made for the model number key in the figures. For calculations 0 through 7 represented in Figure 2, only the densities of layers have

Table 1. Properties of the Media Considered in Figures $2-4$

\begin{tabular}{llllllllll}
\hline $\begin{array}{l}\text { Data } \\
\text { Set }\end{array}$ & $\rho_{1}$ & $\rho_{2}$ & $\rho_{3}$ & $\lambda_{1}$ & $\lambda_{2}$ & $\lambda_{3}$ & $\mu_{1}$ & $\mu_{2}$ & $\mu_{3}$ \\
\hline 0 & \multicolumn{10}{c}{ Homogeneous } & half-space & $\rho=3.0$, & $\lambda=$ & $\mu=3.0$ \\
1 & 3.0 & 3.0 & 3.0 & 3.0 & 3.0 & 3.0 & 3.0 & 3.0 & 3.0 \\
2 & 2.5 & 2.5 & 3.0 & 3.0 & 3.0 & 3.0 & 3.0 & 3.0 & 3.0 \\
3 & 3.0 & 1.5 & 3.0 & 3.0 & 3.0 & 3.0 & 3.0 & 3.0 & 3.0 \\
4 & 3.0 & 6.0 & 3.0 & 3.0 & 3.0 & 3.0 & 3.0 & 3.0 & 3.0 \\
5 & 1.5 & 2.5 & 3.0 & 3.0 & 3.0 & 3.0 & 3.0 & 3.0 & 3.0 \\
6 & 6.0 & 4.5 & 3.0 & 3.0 & 3.0 & 3.0 & 3.0 & 3.0 & 3.0 \\
7 & $D=20$, & $\rho_{1}=2.5, \rho_{2}=3.0$, & $\lambda_{i}=$ & $\mu_{i}=3.0$, & $i=1,2$ \\
8 & 3.0 & 3.0 & 3.0 & 3.0 & 3.0 & 3.0 & 1.0 & 1.0 & 3.0 \\
9 & 3.0 & 3.0 & 3.0 & 3.0 & 3.0 & 3.0 & 3.0 & 0.003 & 3.0 \\
10 & 3.0 & 3.0 & 3.0 & 3.0 & 3.0 & 3.0 & 3.0 & 300.0 & 3.0 \\
11 & 3.0 & 3.0 & 3.0 & 3.0 & 3.0 & 3.0 & 1.0 & 2.0 & 3.0 \\
12 & 3.0 & 3.0 & 3.0 & 3.0 & 3.0 & 3.0 & 9.0 & 6.0 & 3.0 \\
13 & $D=20, \rho_{1}=\rho_{2}=3.0$, & $\mu_{1}=2.5, \mu_{2}=\lambda_{i}=3.0, i=1,2$
\end{tabular}

Equivalents for $\rho$ are $\mathrm{g} / \mathrm{cm}^{3}=10^{3} \mathrm{~kg} / \mathrm{m}^{3}$ and for $\lambda$ and $\mu$ are $10^{11} \mathrm{dyn} / \mathrm{cm}^{2}=10^{12} \mathrm{~N} / \mathrm{m}^{2}$. 

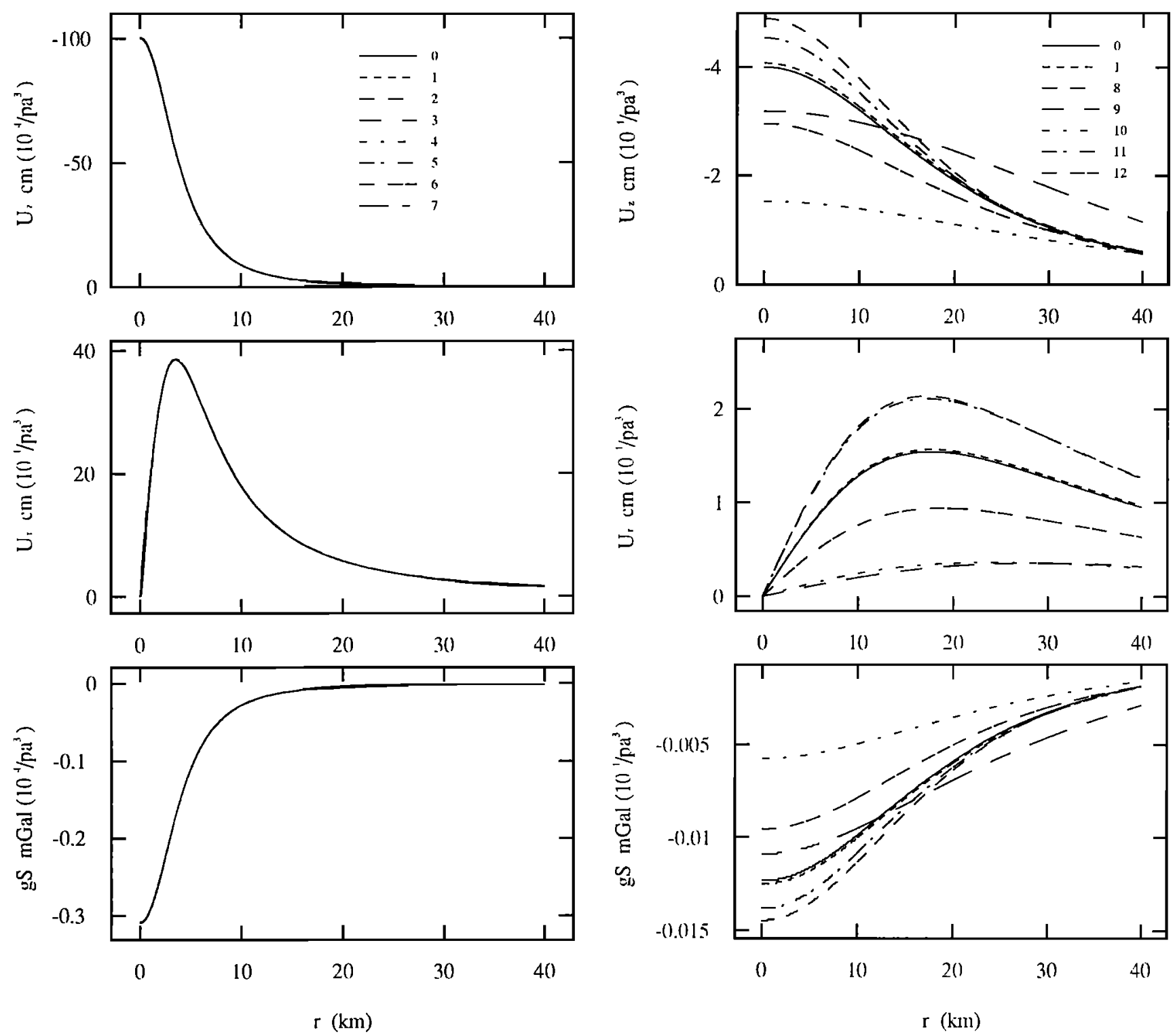

Figure 2. Vertical deformation $U_{z}$, radial deformation $U_{r}$ and surface gravity change $g S$, due to a center of expansion of strength $p a^{3}$, where $p$ is the pressure in bars and $a$ the radius in kilometers, located at $5 \mathrm{~km}$ depth (layer one) in media 0 to 7 described in Table 1 .

been changed. With respect to accuracy, the difference between calculations for the homogeneous half-space, 0 , and the layered half-space with uniform reference densities and uniform elastic moduli, 1 , are of the order of $1 \mu \mathrm{Gal}$ in gravity and $1 \mathrm{~mm}$ in displacement, due to errors in the numerical integration of equations (3) and (4). The difference in accuracy between our results and those of Rundle [1982] are of the same order. We cannot compare radial displacements between our calculations and those of Rundle [1982] because radial displacements were not calculated by Rundle [1982]. It can be seen that changing the reference density in the layers does not significantly change the results.

In Figure 3 we show the same gravity and displacement changes for models 0,1 and 8 to 13 , where only the value of shear modulus in the layers is changed, due to a center of

Figure 3. Same as Figure 2 with the center of expansion located at $25 \mathrm{~km}$ depth (half-space) in media 0,1 , and 8 to 12.

expansion located at $25 \mathrm{~km}$ depth (half-space). Here the differences from the half-space calculation are significant. Figure 4 shows the same quantities due to the presence of a point mass of one mass unit (MU; $1 \mathrm{MU}=10^{15} \mathrm{~g}=10^{12} \mathrm{~kg}$ ). In this case the source is located at $15 \mathrm{~km}$ depth, in layer 2. An important effect of shear modulus change is seen for model 9, which has the source in the middle layer and a very low shear modulus in the middle layer.

\section{Application of the Model to Lanzarote Island}

As an example, we calculate the deformation and gravity change due to a point magma source embedded within the crust beneath a typical oceanic volcanic region. The island of Lanzarote, shown in Figure 5, is the easternmost of the Canary Islands and, with a surface area of $795 \mathrm{~km}^{2}$, the fourth largest. The largest eruption in modern times occurred in the 18th century, in an almost uninterrupted episode that 

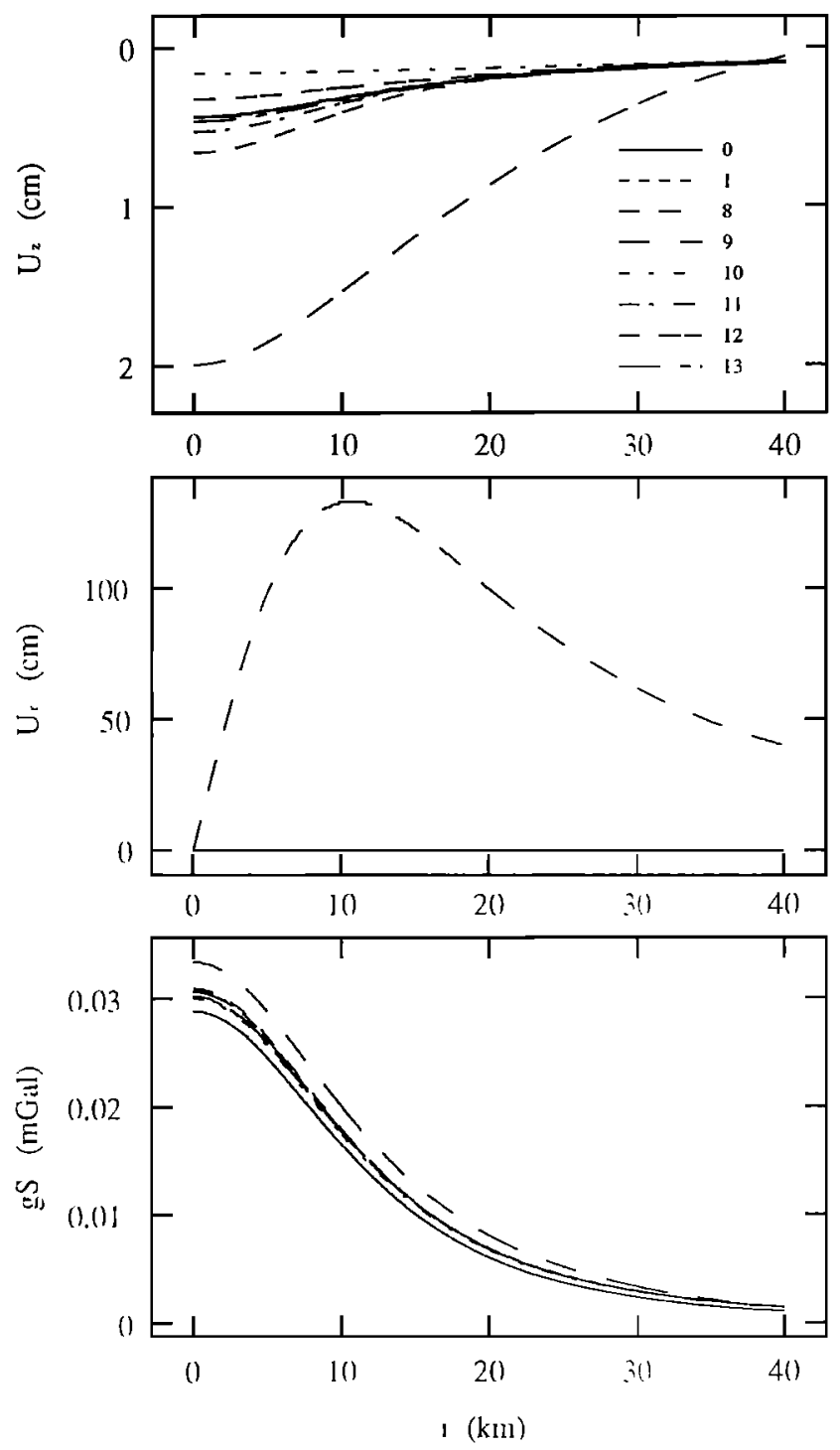

Figure 4. Vertical deformation $U_{z}$, radial deformation $U_{r}$ and surface gravity change $g S$, due to a point mass of $1 \mathrm{MU}$ located at $15 \mathrm{~km}$ depth (layer two) in media 0,1 , and 8 to 13. lasted from 1730 to 1736 . The central part of the island was extensively altered by flows of lava and lapilli covering $172 \pm 5 \mathrm{~km}^{2}$ of land [Ortiz et al., 1986]. Near the center of the eruption zone, where the most important eruptions were recorded, are the so-called Montañas del Fuego or Timanfaya, with over 25 craters within a few square kilometers. The eruptions occurred along a volcano-tectonic fissure, oriented in a WSW-ENE direction, coinciding with the former fissure which in a previous era determined the first eruptions that occurred in the Quaternary period. A period of dormancy extended from 1736 until 1824 terminating with the start of a new phase of activity. Beginning in 1812, a series of small, localized earthquakes preceded an eruption that was much shorter and less important than the previous one, lasting only from July 31 to October 25, 1824 [Rumeu and Araña, 1982].

The characteristics of the island of Lanzarote have led to numerous research programs in the last few decades. Several projects are currently underway, including one being conducted by the Instituto de Astronomía y Geodesia of Madrid, whose objectives include research into the prediction of volcanic risk on the island. Explosion seismic experiments conducted in the Canary Islands in 1977 were utilized to determine a velocity model of the crust of the island of Lanzarote [Banda et al., 1981; Suriñach, 1986]. The model includes three layers, the third being identified with the mantle. As given by Fernández [1992], each layer is assigned elastic properties and densities. The different properties and thicknesses are listed in Table 2. For our purposes, we equate the two-layer crust with the elastic oceanic lithosphere, and the mantle to the asthenosphere.

To illustrate the utility of these calculations for predictive purposes, let us assume that a future volcanic intrusion beneath Timanfaya National Park on Lanzarote has the characteristics of the intrusive episode that resulted in the 1730-1736 eruption sequence. Thus we assume magmatic injection at a depth of $4 \mathrm{~km}$, with a mass of basalt corresponding to a spherical volume with a radius of $0.3 \mathrm{~km}[A-$ raña et al., 1984]. Assume a presure increase of $150 \mathrm{MPa}$, and a density of $2800 \mathrm{Kg} / \mathrm{m}^{3}$, yielding a mass intrusion of $3.210^{11} \mathrm{Kg}$. The resulting deformation and surface gravity changes are shown in Figure 6. The possibility of detecting the effects are as follows.

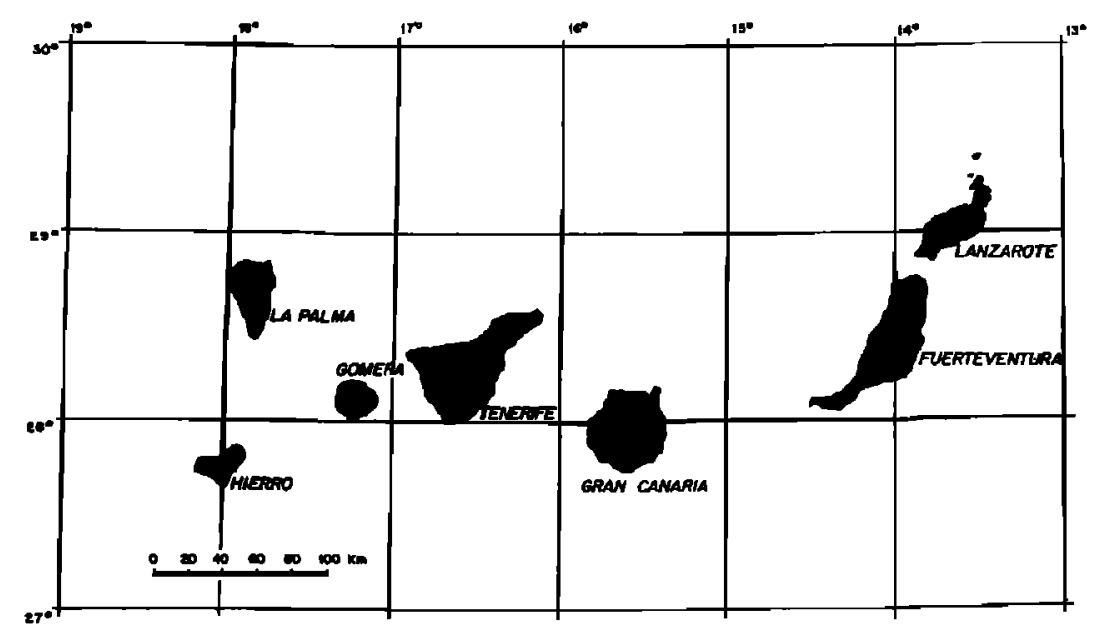

Figure 5. Canary Islands location map. 
Table 2. Densities and Lamé constants for the crustal model of Lanzarote Island

\begin{tabular}{ccccc}
\hline Layer & $\begin{array}{c}\text { Thickness, } \\
\mathrm{km}\end{array}$ & $\begin{array}{c}\rho, \\
\mathrm{g} / \mathrm{cm}^{3}\end{array}$ & $\begin{array}{c}\mu, \\
10^{11} \mathrm{dyn} / \mathrm{cm}^{2}\end{array}$ & $\begin{array}{c}\lambda, \\
10^{11} \mathrm{dyn} / \mathrm{cm}^{2}\end{array}$ \\
\hline 1 & 4.0 & 2.2 & 1.065 & 1.390 \\
2 & $7-8$ & 2.7 & 2.940 & 3.519 \\
Mantle & & 3.1 & 5.211 & 6.554
\end{tabular}

Data are from Fernández [1992].

Vertical Deformation

The maximum vertical deformation in this case has an absolute value of $3.1 \mathrm{~cm}$, causing a tilt capable of detection anywhere on the island, and in particular at the Geodynamic Station located in the north part of the island [Vieira et al., 1991]. With levelling profiles and geodetic networks having radial dimensions of $3 \mathrm{~km}$ over the intrusion, detection of the vertical displacement is very possible.

Gravity Changes

Surface gravity changes are greater than $10 \mu \mathrm{Gal}$ up to a surface distance of almost $9 \mathrm{~km}$ from the intrusion, hence these can readily be detected by microgravity networks in the area and using permanently recording relative gravimeters. Absolute gravimeters can also detect the gravity changes within radial distances up to $20 \mathrm{~km}$.
Horizontal Deformations

Horizontal deformations can be detected reobserving geodetic networks using classical methods up to a maximun radial distance of $5 \mathrm{~km}$. Measurements made with a $10-\mathrm{m}-$ long horizontal extensometer could be used to detect deformation at stations $30 \mathrm{~km}$ away. If a $50-\mathrm{m}$ instrument is used, effects can be detectes up to radial distances of $50 \mathrm{~km}$. Thus horizontal deformation could be observed using the 48-m horizontal extensometer at the the Geodynamic Station of Lanzarote [Fernández et al., 1993].

\section{Conclusions}

We have extended earlier methods to compute the displacements and gravity changes due to a point magma intrusion in layered elastic-gravitational media. Specifically, our method can be used for models in which the source can be placed at arbitrary depth in a medium consisting of two layers overlying a half-space. However, the numerical technique can be easily generalized to a medium consisting of an arbitrary number of layers overlying a half-space. The same general methods can also be used to calculate stresses and potential changes as well. These methods would be particularly valuable in interpreting changes in tide gauges following eruption of a volcanic island, because tide gauges measure a combination of changes in uplift and gravitational potential.

(a) Vertical Deformation $\mathrm{cm}$.

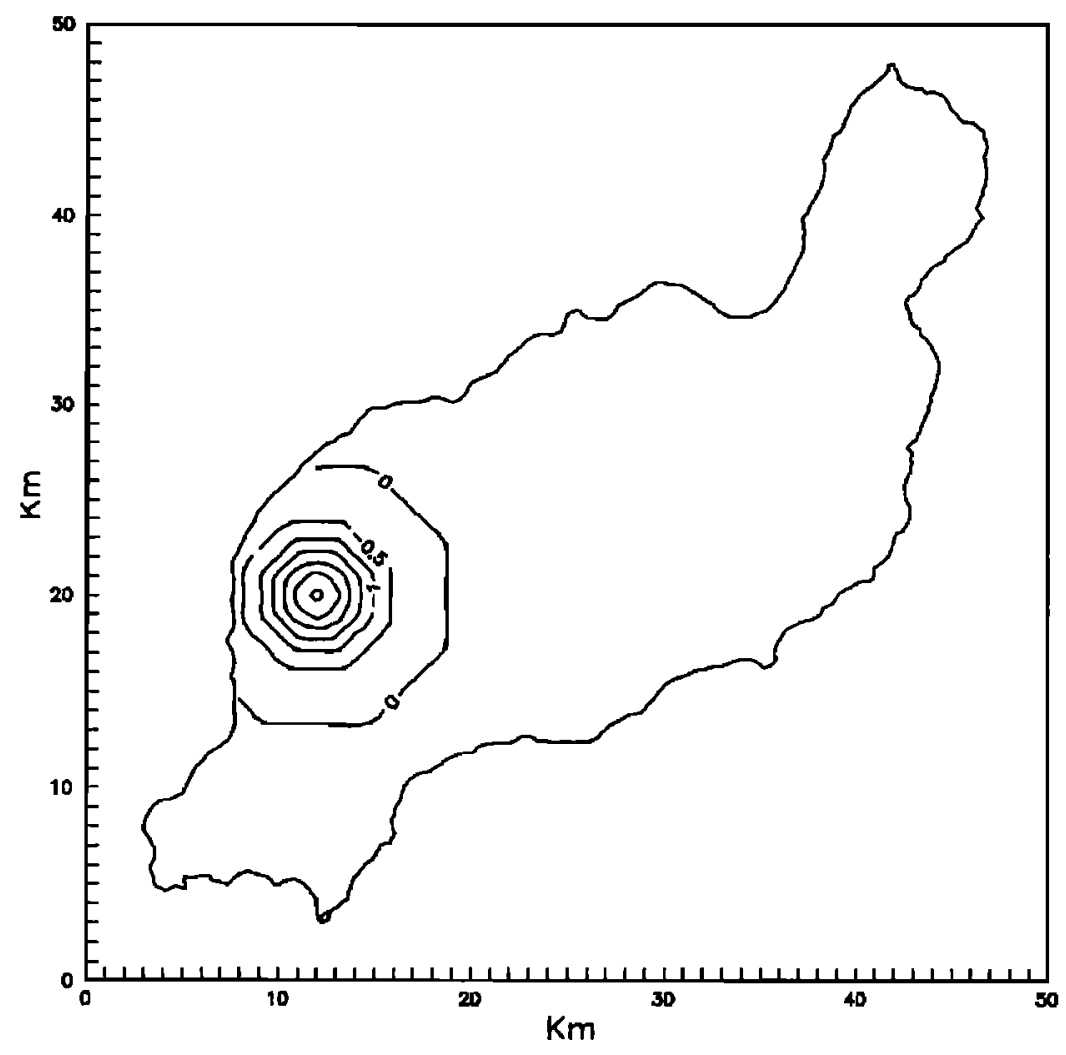

Figure 6. (a) Vertical deformation in centimeters, (b) surface gravity variation in $\mu$ Gal, and (c) radial deformation in centimeters due to a magma intrusion of $0.3 \mathrm{~km}$ radius located $4 \mathrm{~km}$ below the Timanfaya National Park. A pressure increase of $150 \mathrm{MPa}$ and a mass of $3.210^{11} \mathrm{Kg}$ are assumed. 
(b) Surface Gravity Change

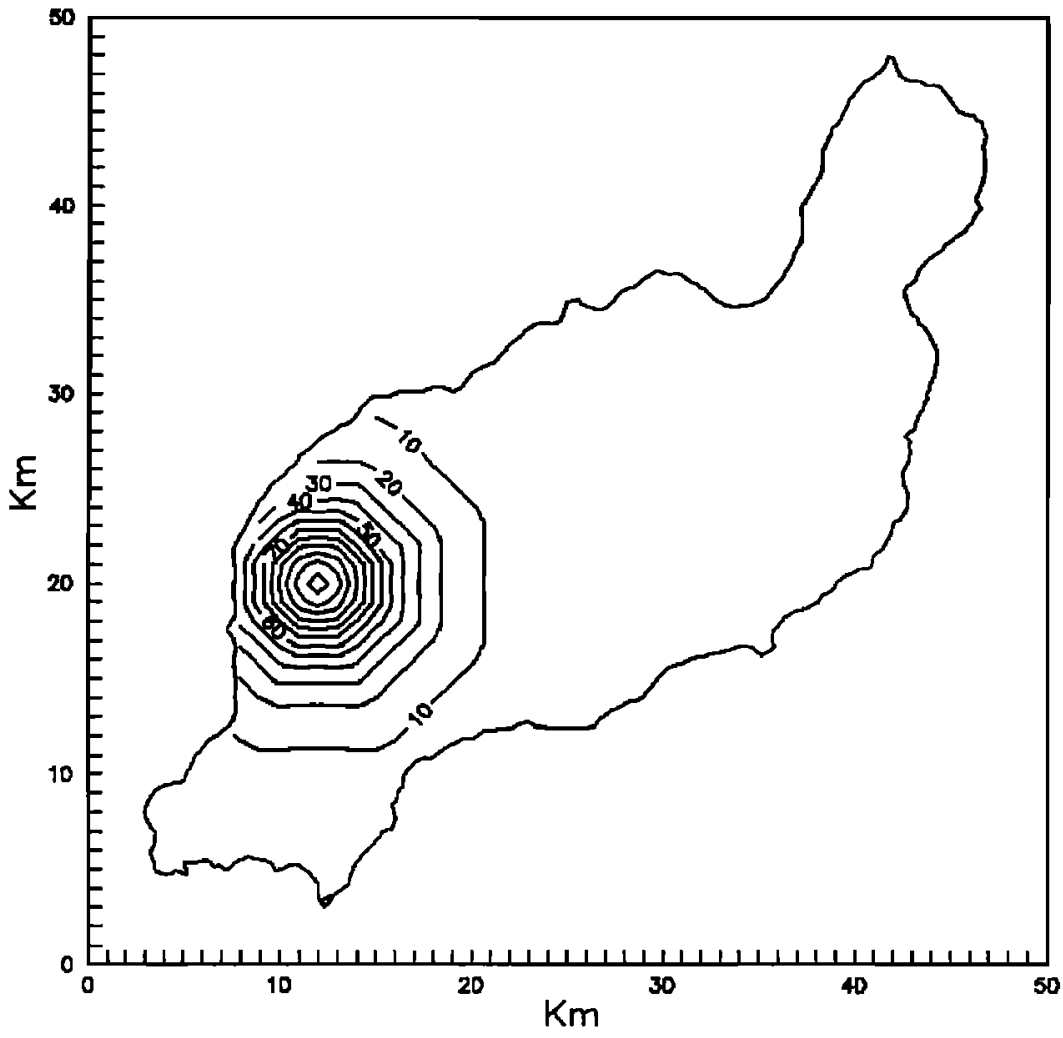

(c) Radial Deformation $\mathrm{cm}$.

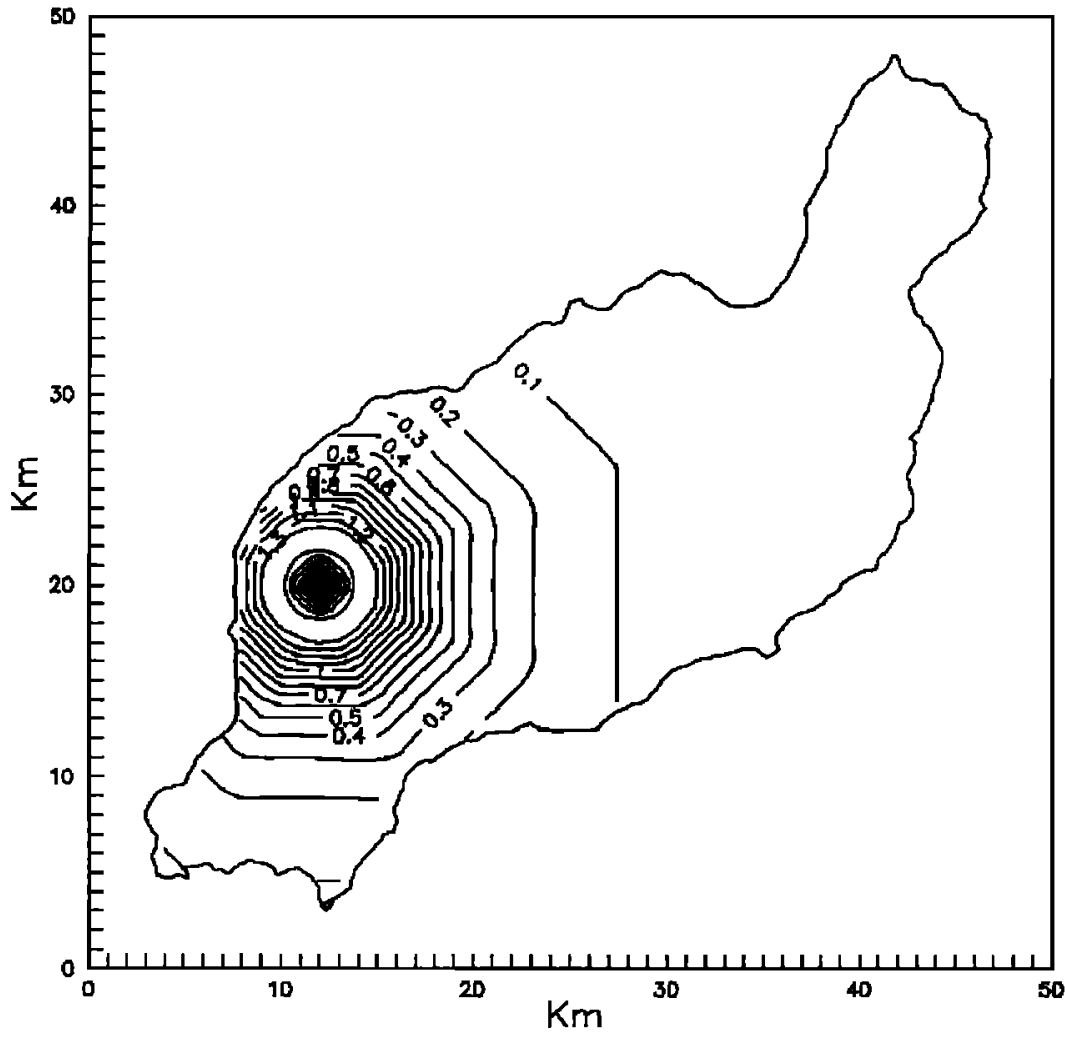

Figure 6. (continued) 
Our results indicate that variations in elastic moduli have a much more significant effect than variations in reference density on both the surface displacements and gravity changes. Deviations of the half-space displacements in the layered medium from the homogeneous half-space are clearly due to variations in strain arising from changes in the elastic moduli. However, variations in surface gravity are also influenced far more by changes in moduli than by changes in density, implying that changes in density due to volumetric dilatation are significantly larger than Bouguer effects caused by changing the layer reference densities. Our results clearly indicate that models with uniform density and moduli, or even a single layer over a half-space, may be too simple to adequately model observed gravity and deformation changes in volcanic regions. Alternatively, our calculations give a more detailed representation of plate flexure in the near region than the thin plate calculations discussed elsewhere in the literature.

The deformation, gravity, and potential changes due to point sources can be used as Green's functions in more realistic calculations involving volumetrically distributed sources. Moreover, patterns of gravity and deformation changes due to magmatic intrusion into a volcanically active area such as Lanzarote island can be predicted on the basis of past activity, leading to the possiblity of early detection and hazard mitigation. Any observed patterns of deformation can be used to compute the probability that an eruption may occur, that is, in risk assessment calculations [e.g., Sartoris et al., 1990]. Finally, the same methods developed here can be used with other source functions [see Rundle, 1980] to calculate deformation and gravity changes from earthquake faulting in models with several layers overlying a half-space.

Acknowledgments. The research of J.F. was supported with funds from project PB88-0022 (DGICYT) conducted in the Instituto de Astronomía y Geodesia, and under the auspices of the U.S. Department of Energy at Lawrence Livermore National Laboratory under contract W-7405-ENG-48. The research of J.B.R. was supported under the auspices of the U.S. Department of Energy at Lawrence Livermore National Laboratory under contract W-7405-ENG-48, Office of Basic Energy Sciences, Division of Geosciences. We are grateful for comments on previous versions of this paper by $R$. Vieira, J. L. Díez, and G. Díaz, and to S. Larsen for his help with computer graphics.

\section{References}

Araña, V., J.L. Díez-Gil, R. Ortíz, and J. Yuguero, Convection of geothermal fluids in the Timanfaya volcanic area (Lanzarote, Canary Islands), Bull. Volcanol., 47, 667-677, 1984.
Banda, E., J.J. Dañobeitia, E. Suriñach, and J. Ansorge, Features of crustal structure under the Canary Islands, Earth Planet. Sci. Lett., 55, 11-24, 1981.

Fernández, J., Técnicas geodésicas y geodinámicas aplicadas a la investigación del riesgo volcánico en la isla de Lanzarote. Ph.D. thesis, 149 pp., Universidad Complutense de Madrid, 1992.

Fernández, J., J. Arnoso, and R. Vieira, Investigación en riesgo volcánico en Lanzarote, Rev. Ac. CC. Ex. Fís. Nat. de Madrid, in press, 1993.

Gilbert, F., and G. Backus, Propagator matrices in elastic wave and vibration problems, Geophysics, 31, 326-332, 1966.

Haskell, N.A., The dispersion of surface waves on multilayered media, Bull. Seismol. Soc. Am., 43, 421-440, 1953.

Love, A.E.H., Some Problems in Geodynamics, 180 pp., Cambridge University Press, New York, 1911.

Ortíz, R., V. Araña, and C. Valverde, Aproximación al conocimiento del mecanismo de la erupción de 1730-1736 en Lanzarote, An. Fís., Ser. B, 82, 127-142, 1986.

Rumeu, A., and V. Araña, Diario pormenorizado de la erupción volcánica de Lanzarote en 1824, Anu. Estud. Atl., 28 , 15-61, 1982.

Rundle, J.B., Viscoelastic crustal deformation of a layered half-space by point couple sources, J. Geophys. Res., 83, 5937-5945, 1978.

Rundle, J.B., Static elastic-gravitational deformation of a layered half-space by point couple sources, J. Geophys. Res., 85, 5355-5363, 1980.

Rundle, J.B., Numerical evaluation of static elastic gravitational deformation in a layered half-space by point couple sources, Rep. 81-2058, Sandia Nat. Lab., Albuquerque, N.M., 1981.

Rundle, J.B., Deformation, gravity, and potential changes due to volcanic loading of the crust, J. Geophys. Res., 87, 10729-10744, 1982. (Correction, J. Geophys. Res., 88, 10647-10652, 1983.)

Sartoris, G., J.P. Pozzi, C. Philippe, and J.L. Le Motel, Mechanical stability of shallow magma chambers, J. Geophys. Res., 95, 5141-5151, 1990.

Singh, S.J., Static deformation of a multilayered half space by internal sources, J. Geophys. Res., 75, 3257-3263, 1970.

Suriñach, E., La estructura cortical del Archipiélago Canario. Resultados de la interpretación de perfiles sísmicos profundos, An. Fis., Ser. B, 82, 62-77, 1986.

Thomson, W.T., Transmission of elastic waves throught a stratified medium, J. Appl. Phys., 21, 89-93, 1950.

Vieira, R., M. Van Ruymbeke, J. Fernández, J. Arnoso, and C. Toro, The Lanzarote underground laboratory, Cah. Cent. Eur. Géodyn. Séismol., 4, 71-86, 1991.

J. Fernández, Instituto de Astronomía y Geodesia, Facultad de Ciencias Matemáticas, Ciudad Universitaria, 28040-Madrid, Spain.

J. B. Rundle, Cooperative Institute for Research in Environmental Sciences, University of Colorado, Boulder, CO 80309.

(Received February 10, 1993; revised July 26, 1993; accepted August 26, 1993.) 